\title{
AVAILABILITY OF EXCHANGEABLE AND NON-EXCHANGEABLE POTASSIUM IN SOME JORDANIAN CALCAREOUS SOILS
}

(Received: 24.9.2009)

\author{
By \\ F. M. Al-Nasir \\ Department of Plant Production, Faculty of Agriculture, Mutah University, Al-Karak, Jordan
}

\begin{abstract}
This study aimed at estimating the quantity of exchangeable and non-exchangeable potassium (K) in calcareous agricultural soils in Jordan, and determining the contribution of exchangeable and non-exchangeable $\mathrm{K}$ to plant nutrition. The soils were collected from 18 different agricultural regions, and thereafter cultivated with alfalfa without adding fertilizers. The results showed significant differences for dry matter, total $\mathrm{K}$ uptake of the plant, and in the contribution of exchangeable and non-exchangeable $\mathrm{K}$ in different soils. The soils were divided into two major groups. The first group included Deer Alah, Alzaitunah, Almafrag, Alshubak and Albagah, which characterized mainly by high $\mathrm{K}$ in vegetative parts and optimum soil K content enough for Alfalfa growth in the second and third cuts. The other soils were clustered together in one group, which showed lower $\mathrm{K}$ concentrations than the optimum. Dry matter production was significantly correlated with $\mathrm{K}$ uptake in plant tissue $\left(\mathrm{r}=0.755^{* *}\right)$, and with exchangeable and non-exchangeable $\mathrm{K}\left(\mathrm{r}=0.536^{*}\right.$ and $0.589^{*}$, respectively). Total $\mathrm{K}$ uptake was significantly correlated with initial exchangeable $\mathrm{K}$, and the contribution of exchangeable and non-exchangeable $\mathrm{K}$ in plant nutrition.
\end{abstract}

Key words: alfalfa, calcareous soil, soluble $K$, exchangeable $K$, non-exchangeable $K$, total $K$ uptake.

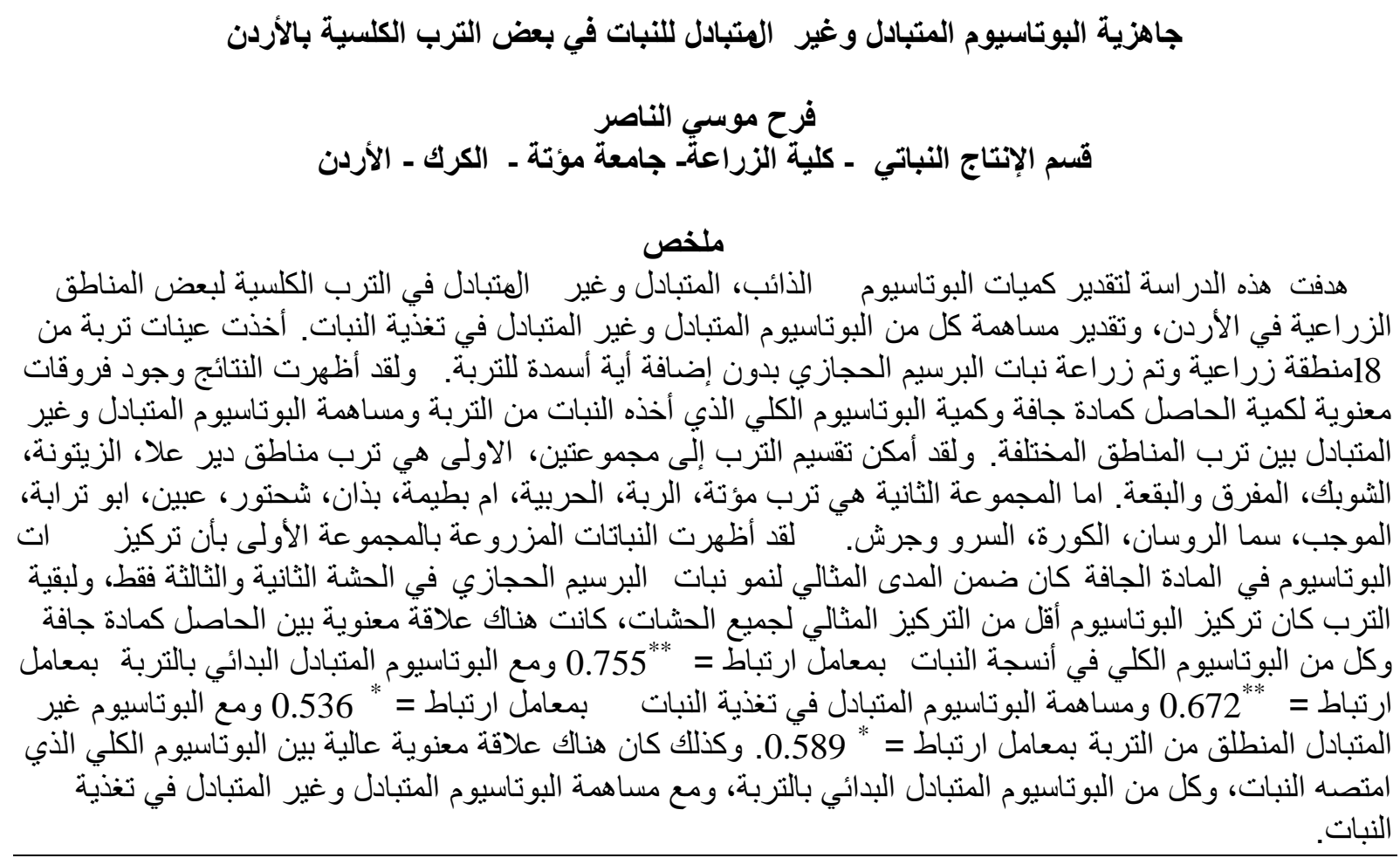


البوتاسيوم غير المتبادل و المنطلق من الترب بةً بالتسميد

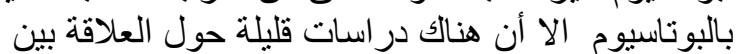

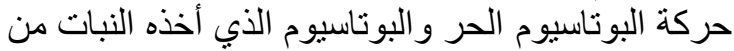
الترب الكلسية (Jalali, 2006).

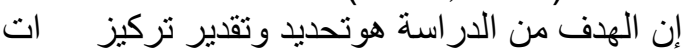

Soluble, البوتاسيوم الذائب المنبادل وغير النتبادل exchangeable and nonexchengeable K الترب الكلسية في بعض المناطق الهامة زر اعياً بالأردن،

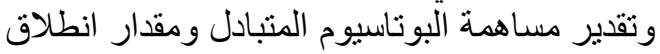

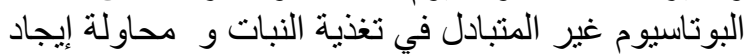

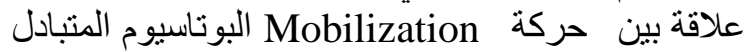
و غير المتبادل مع بعض صنفات التربة.

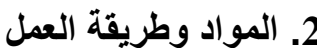

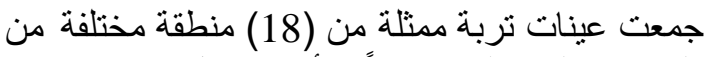

أهم المناطق المستغلة زر اعياً بالأردن شكل رقم (1) منطة

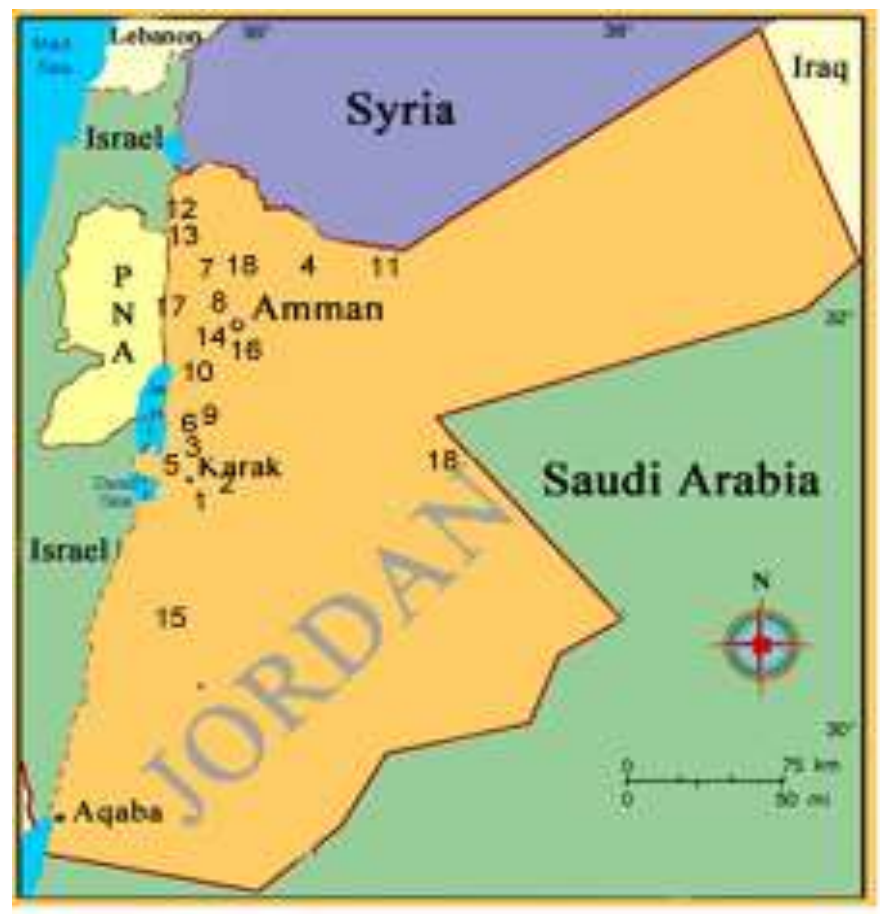

شكل (1) خارطة تبين مواقع جمع العينات. جدول 1 يبين اسماء

المواقع

Fig. (1): Collection sites of soil samples. See Table 1 for collection site names.

و التي تتباين من حيث الصفات وقد تم نخل العينات

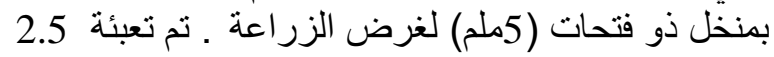

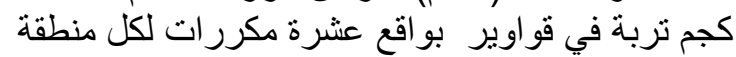

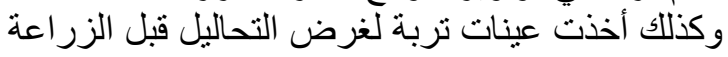

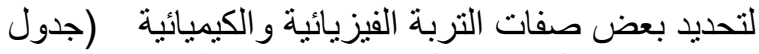

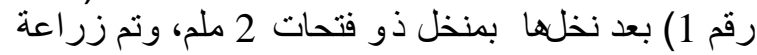
15 بذرة من نبات في كل قارورة بتاريخ (sativa L. Medicago)

\section{1. \\ يعتبر البوتاسيوم عنصر غذائي أساسي لنمو النبات}

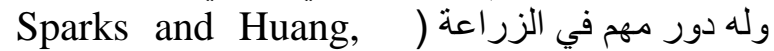
1985)، وبشكل عام فأن ترب الزب المناطق الجافة وشبه

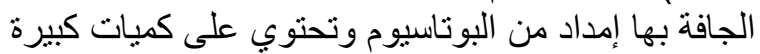

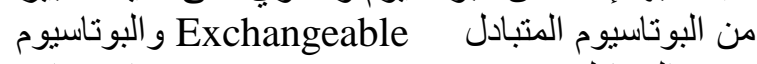
غير الزتبادل Non-exchangeable و عند استخدام نظام الزراعة الكثيفة فأن هذة الترب تعاني من نقص في

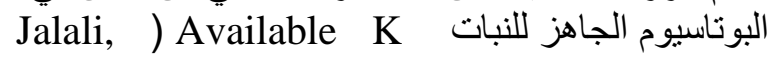

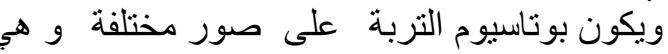

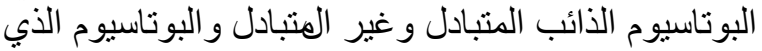

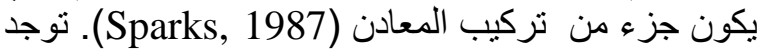

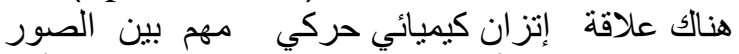

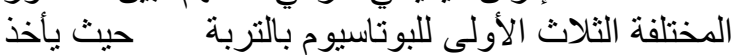

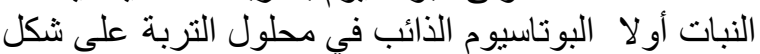

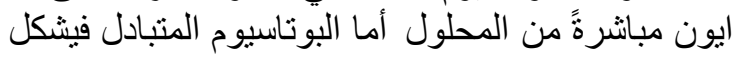

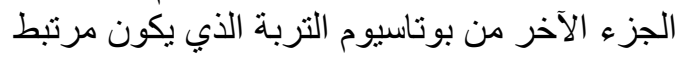

بشحنة كهربائية على سطوح غرويات التئي التربة المعدنية

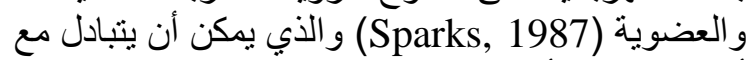

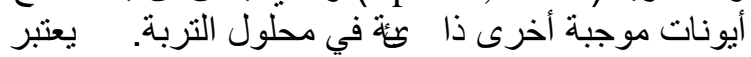

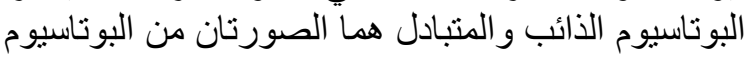

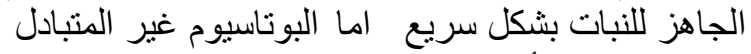

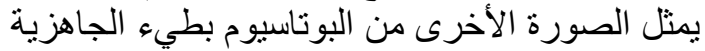
للنبات و الذي يشكل احتياطي هام للبوتناسيوم فيطي في التربة التزبة

.(Jalali, 2006)

لقد أوضحت دراسات عديدة بأن البوتاسيوم غير

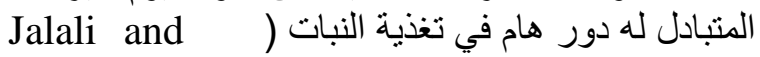
Zarabi, 2006; and Mengel and (Uhlenbecker, 1993

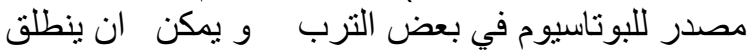

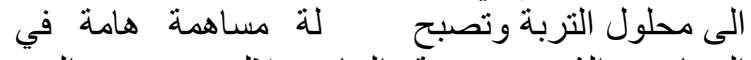

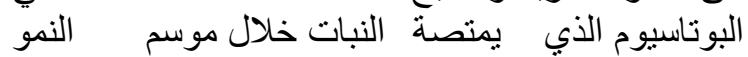
Rahmatulla et al., ) ويستخدم خلال أيام قليلة . (1994; and Øgaard and Krogstad, 2005 يساهم البوتاسيوم غير المتبادل مساهمة هامة" في تغذية

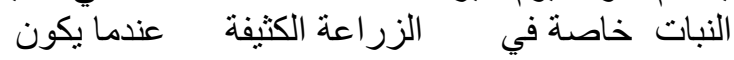
البوتاسيوم المتبادل غير كافي لسد احتياجات النبات (Binet et al., 1984; and Mengel, 1985) حالة تناقص مستوى البوتاسيوم الذائب و المتبادل إلى حد

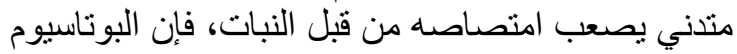

غير المنبادل ينطلق من بين طبقات المعادن الطينية (Maclean and Watson, 1985) بها (1990) Dhillon and Dhillon وجدا أنه عندما يتناقص مستوى البوتاسيوم الذائب و المنبادل

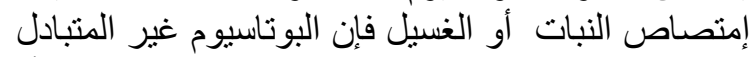

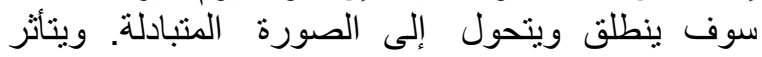




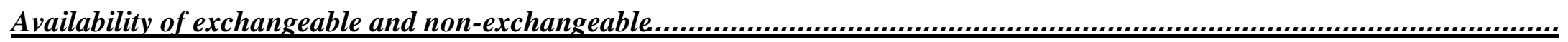

Table (1): Some chemical and physical properties of studied soils.

\begin{tabular}{|c|c|c|c|c|c|c|c|c|c|c|c|c|c|}
\hline \multirow[t]{2}{*}{ المنطقة } & \multirow[t]{2}{*}{ Location } & \multirow[t]{2}{*}{$\begin{array}{l}\text { Soil } \\
\text { No. }\end{array}$} & \multicolumn{3}{|c|}{$\begin{array}{c}\text { Particle size distribution } \\
(\%)\end{array}$} & \multirow[t]{2}{*}{ Texture } & \multirow[t]{2}{*}{$\begin{array}{c}\text { pH } \\
(1: 1)\end{array}$} & \multirow{2}{*}{$\begin{array}{c}\mathrm{EC} \\
(1: 1 \\
\left.\mathrm{ms} \mathrm{cm}^{-1}\right)\end{array}$} & \multirow[t]{2}{*}{$\begin{array}{l}\text { O.M. } \\
(\%)\end{array}$} & \multirow[t]{2}{*}{$\begin{array}{c}\mathrm{CaCO}_{3} \\
(\%)\end{array}$} & \multirow{2}{*}{$\begin{array}{c}\text { CEC } \\
\left(\mathrm{meq} 100 \mathrm{~g}^{-1}\right. \\
\text { soi) }\end{array}$} & \multirow{2}{*}{$\begin{array}{l}\text { Avail. K } \\
\left(\mathrm{mg} \mathrm{kg}^{-1}\right. \\
\text { soil) }\end{array}$} & \multirow[t]{2}{*}{$\begin{array}{c}\text { Avail. } P \\
\left(\mathrm{mgkg}^{-1} \text { soil }\right)\end{array}$} \\
\hline & & & Silt & Sand & Clay & & & & & & & & \\
\hline مؤتة & Mutah & 1 & 11.5 & 69 & 19.5 & s. 1. & 7.86 & 0.6 & 1.08 & 19 & 42 & 282.4 & 24.6 \\
\hline الحربية & Alharbih & 2 & 12 & 64.5 & 23.5 & s. c. 1. & 7.8 & 0.5 & 0.87 & 29 & 25 & 320.2 & 71.8 \\
\hline الربة & Alrabah & 3 & 14 & 58.5 & 27.5 & s. c. l. & 7.8 & 0.6 & 1.26 & 24 & 40 & 464.2 & 76.4 \\
\hline ام بطيمة & um abtamah & 4 & 11.5 & 65 & 23.5 & s. c. 1. & 7.63 & 0.6 & 1.5 & 23 & 32 & 376.2 & 70.8 \\
\hline بذان & Bathan & 5 & 4 & 86.5 & 9.5 & l. S. & 7.63 & 1.8 & 1.68 & 21 & 28 & 394.1 & 68 \\
\hline شحتور & Shahtur & 6 & 25 & 49 & 26 & s. c. l. & 7.53 & 0.9 & 0.98 & 17.9 & 43 & 458.5 & 51.1 \\
\hline عبين & Abien & 7 & 5.5 & 71 & 23.5 & s. c. l. & 7.46 & 0.6 & 1.6 & 11 & 50.1 & 437.8 & 47.5 \\
\hline البقعة & Albagah & 8 & 15 & 61.5 & 23.5 & S. c. l. & 7.69 & 0.7 & 1.6 & 22 & 55.8 & 463.3 & 67 \\
\hline ابو ترابة & Abu Trabah & 9 & 7.5 & 62 & 24.5 & s. c. l. & 7.88 & 1.1 & 0.84 & 20 & 40.2 & 303.5 & 8.4 \\
\hline الموجب & Almugab & 10 & 17.5 & 64 & 18.5 & s. l. & 7.64 & 0.8 & 0.7 & 31 & 39.2 & 452.1 & 76.4 \\
\hline المفرق & Almafrag & 11 & 18 & 66.5 & 15.5 & s. 1. & 7.06 & 1.6 & 1.3 & 28 & 42.8 & 605.6 & 112 \\
\hline سما & Sama Alrusan & 12 & 8 & 62 & 30 & s. c. 1 . & 7.6 & 0.8 & 1.82 & 16 & 55 & 512.4 & 55.9 \\
\hline الكورة & Alkurah & 13 & 7.5 & 68 & 24.5 & S. c. 1. & 7.15 & 0.9 & 2 & 14 & 57 & 393.4 & 162.8 \\
\hline السرو & Alsuraw & 14 & 9 & 63 & 28 & s. c. l. & 7.46 & 0.7 & 1.2 & 17 & 51.3 & 134.0 & 37 \\
\hline الشوبك & Alshubak & 15 & 9.5 & 74 & 16.5 & s. 1. & 7.49 & 1.2 & 2.1 & 23.3 & 30.2 & 690.5 & 119.3 \\
\hline الزيتونة & Alzaitunah & 16 & 11 & 59.5 & 29.5 & s. c. 1. & 7.55 & 1.7 & 1.82 & 21 & 53 & 501.2 & 101 \\
\hline دير علا & Deer Alah & 17 & 12 & 62.5 & 25.5 & s. c. 1. & 7.74 & 1.8 & 1.64 & 22 & 56 & 856.3 & 104 \\
\hline جرش & Jarash & 18 & 13.5 & 52 & 34.5 & s. c. l. & 7.86 & 0.8 & 1.2 & 20 & 58.5 & 503.4 & 133.3 \\
\hline
\end{tabular}

s. c. 1 = sandy clay loam

$\mathrm{O} \cdot \mathrm{M}=$ Organic matter 
Havlin and Westfall, ) يمن حسابه في معادلة :(1985

Non-exchangeable K release $=\mathrm{C}-(\mathrm{B}-\mathrm{A})$ حيث A و B مستوى البوتاسيوم المتبادل قبل وبعد إجر اء

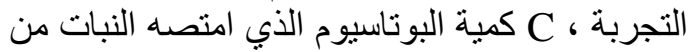

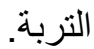

\section{3. النتائسج (2)}

يوضح الجدول رقم (2) كمية الحاصل كمادة جافة

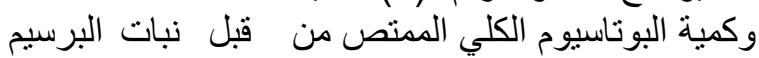
Alfalfa

Non- وغير المتبادل الئبادل Exchangeable K exchangeable K أن أعلى حاصل كمادة جافة كان لتربة منطقة الزيتونة

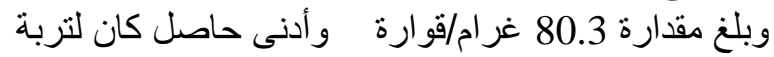

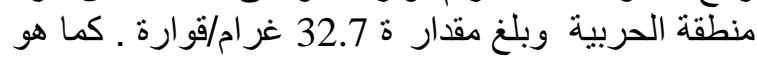

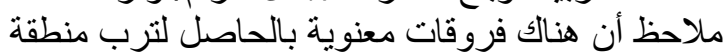

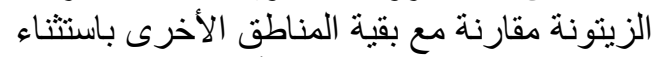

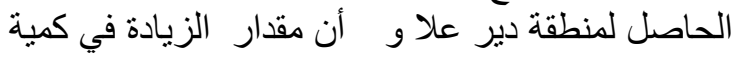

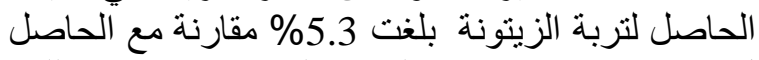

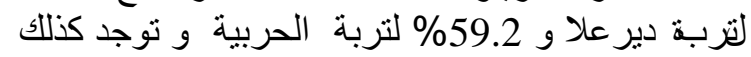

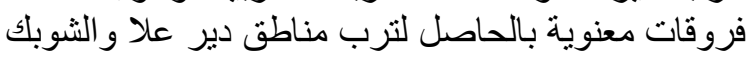

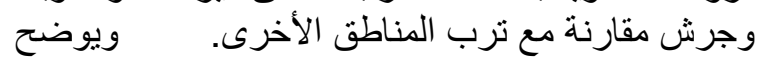

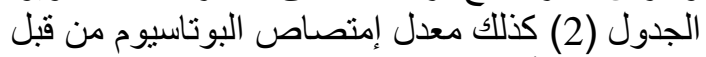

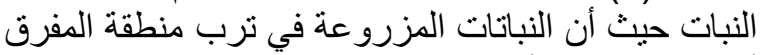

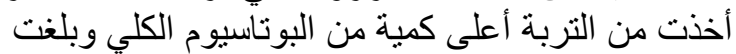

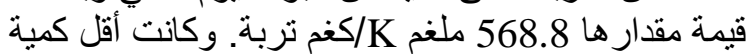
امتصنها النباتات المزرو

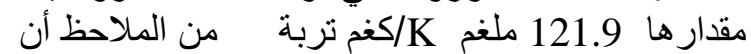
الامتصاص للبوتاسيوم من قبل نبات

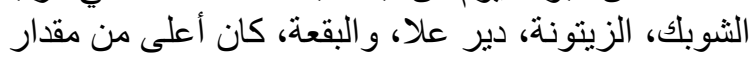

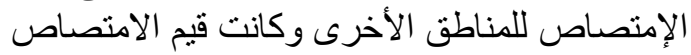

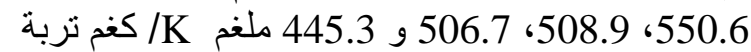

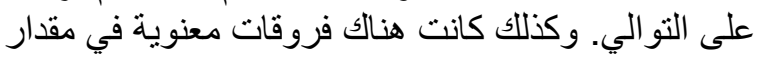

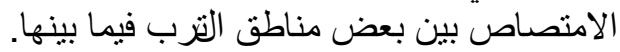

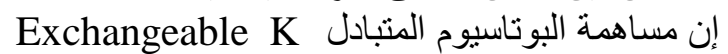

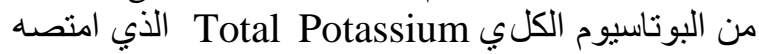

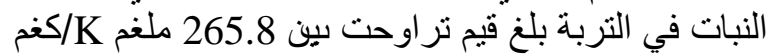

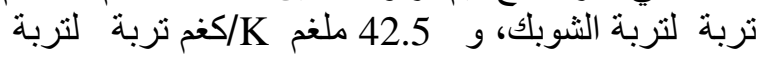

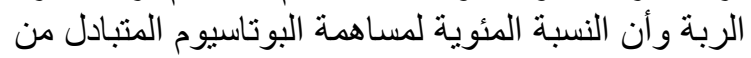

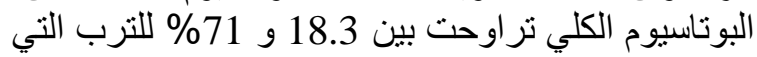

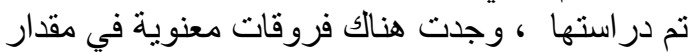

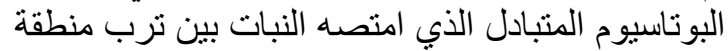

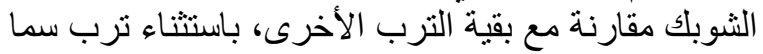

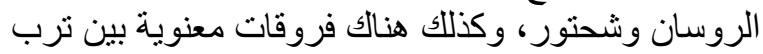

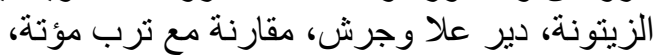

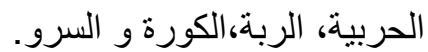

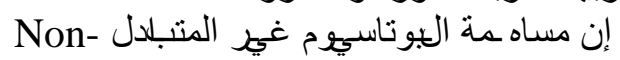

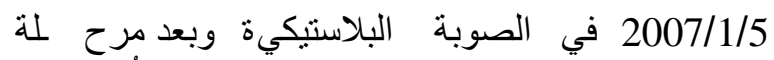

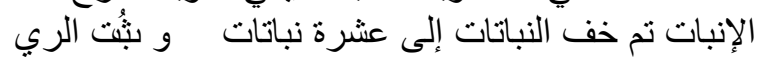

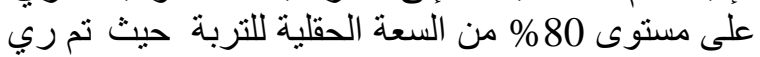

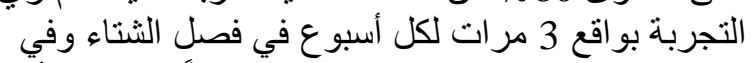

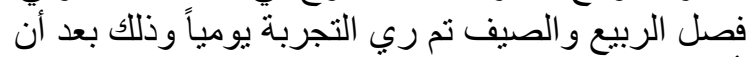

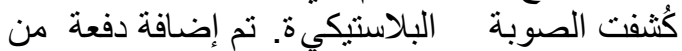

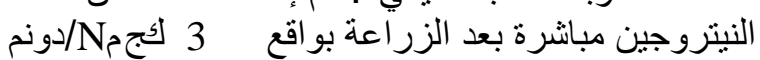
على شكل نترات Starter

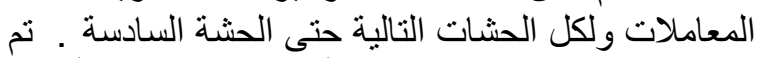

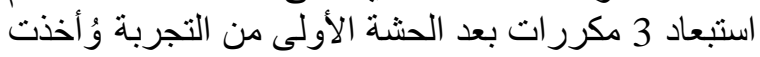

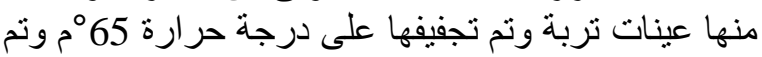

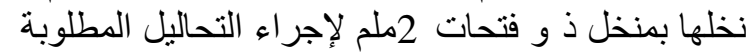
وبعد الحشة الثنانية نم استبعاد 3

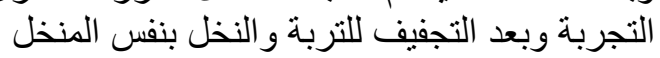

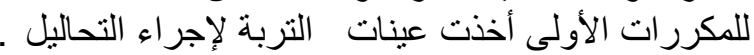

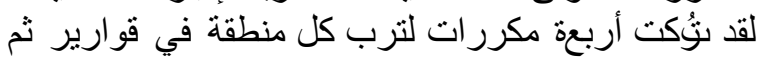

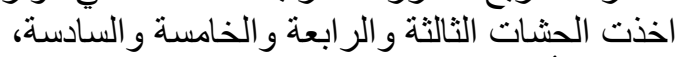

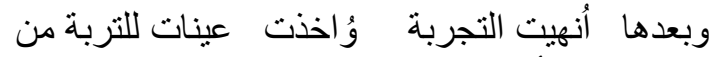

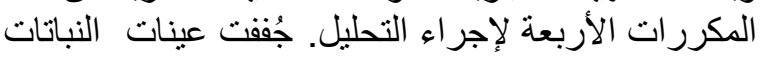

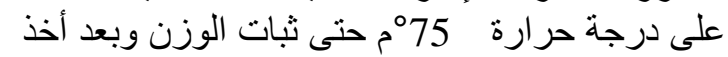

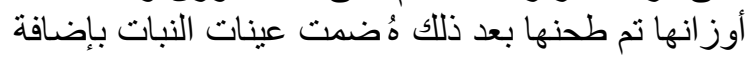
حامض النيتريك 160م. تم تقدير البوتاسيوم بالنبات باستخدام جهاز

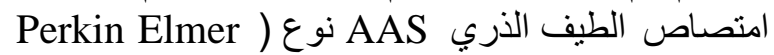

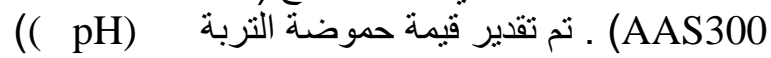

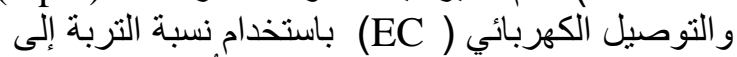

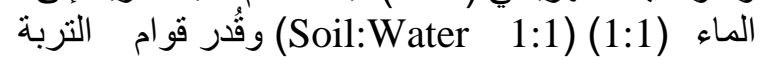

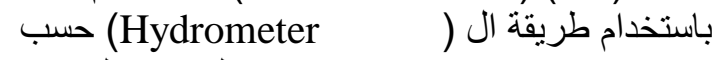
(Bouyoucos, 1951)

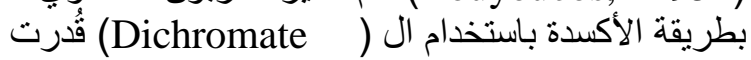

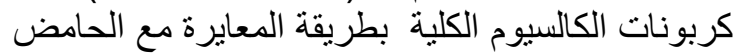

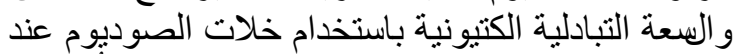

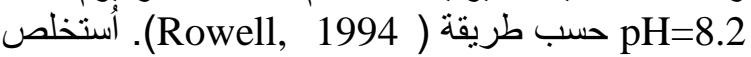

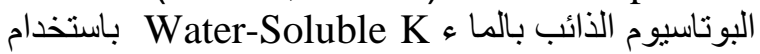
نسبة التربة إلى الماء (1:5) (Soil:Water 1:5) بالرجاء

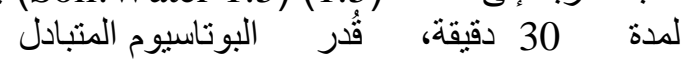
Exchangeable K

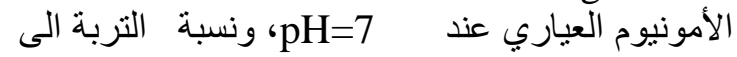

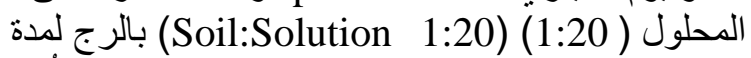

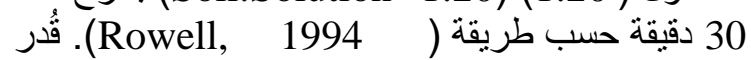
Non-exchangeable K البوتاسيوم غير المتبادل

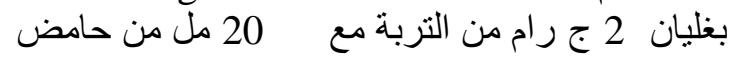
النيتريك العياري طريقة ( Cox et al., 1999) . تم حساب كمية

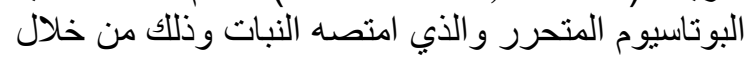

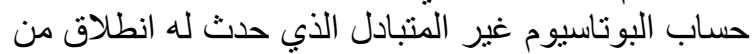

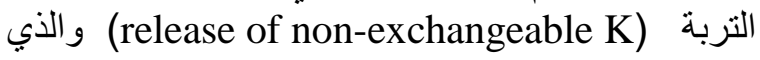


Table (2): Availability of exchangeable $\mathrm{K}$ and non-exchangeable $\mathrm{K}$, cumulative uptake and dry matter.

\begin{tabular}{|c|c|c|c|c|c|c|c|}
\hline \multirow[t]{2}{*}{ المنطقة } & \multirow[t]{2}{*}{ Location } & \multirow[t]{2}{*}{$\begin{array}{l}\text { Soil } \\
\text { No. }\end{array}$} & \multirow[t]{2}{*}{$\begin{array}{l}\text { Dry matter } \\
\text { mg pot }^{-1}\end{array}$} & \multirow[t]{2}{*}{$\begin{array}{l}\text { Dry matter } \\
\text { mg pot }^{-1}\end{array}$} & Total K uptake & Exchan. K uptake & $\begin{array}{c}\text { Non-Excha. } \mathrm{K} \\
\text { uptake }\end{array}$ \\
\hline & & & & & \multicolumn{3}{|c|}{$\mathrm{mg} \mathrm{kg}^{-1}$ soil } \\
\hline مؤتة & Mutah & 1 & 36.9hi & 484.2ji & 193.6ij & $51.8 \mathrm{~g}$ & 141.8efgh \\
\hline الحربية & alharbih & 2 & $32.7 i$ & $347.4 \mathrm{jk}$ & 138.9jk & 43.9g & 95.0ghi \\
\hline الربة & alrabah & 3 & 40.4fghi & 523.3hi & 209.3hi & 42.5g & 166.8ef \\
\hline ام بطيمة & um abtamah & 4 & 47.9defg & 650.7fgh & 260.3fgh & 97.9efg & 162.3ef \\
\hline بذان & Bathan & 5 & 51.5def & 892.4d & 356.9d & 108.1defg & 249.0d \\
\hline شحتور & Shahtur & 6 & 41.5efghi & 730.1ef & 292.0ef & 204.4acb & 87.6hi \\
\hline عبين & Abien & 7 & 47.6defgh & 690.6fg & 276.3fg & 92.2efg & 184.0e \\
\hline البقعة & Albagah & 8 & 49.3defg & 1113.3d & $445.3 c$ & 89.3efg & 355.9b \\
\hline ابو ترابة & Abu Trabah & 9 & 38.7ghi & 501.3hij & 200.5hij & $77.7 \mathrm{fg}$ & 122.8fgh \\
\hline الموجب & Almugab & 10 & 39.2ghi & 568.6ghi & 227.4ghi & 104.5defg & 131.9efgh \\
\hline المفرق & Almafrag & 11 & 52.1de & $1422 a$ & $568.8 a$ & 104.5defg & $464.2 \mathrm{a}$ \\
\hline سما الروسان & Sama Alrusan & 12 & 49.7defg & 872.6de & 349.0de & 248.1ab & 101.1ghi \\
\hline الكورة & Alkurah & 13 & $54.7 \mathrm{~cd}$ & 594.6fghi & 237.8fghi & $53.7 \mathrm{fg}$ & $184.2 \mathrm{e}$ \\
\hline السرو & Alsuraw & 14 & 43.9defghi & $304.9 \mathrm{k}$ & 121.9k & 67.6fg & $54.3 i$ \\
\hline الثوبك & Alshubak & 15 & 65.1bc & 1376.7ab & $550.6 \mathrm{ab}$ & $265.8 \mathrm{a}$ & $290.8 \mathrm{~cd}$ \\
\hline الزيتونة & Alzaitunah & 16 & $80.3 a$ & 1272.4ab & 508.9ab & 166.4cde & 342.7bc \\
\hline دير علا & Deer Alah & 17 & 76.0ab & $1266.8 \mathrm{cb}$ & $506.7 \mathrm{cb}$ & 179.6bcd & 330.1bc \\
\hline جرش & Jarash & 18 & 67.1b & $704.3 f g$ & $281.7 f g$ & 132.1cdef & 149.5efg \\
\hline
\end{tabular}


معنوية لنركيز البوتاسيوم في نباتات منطقتي الزيتونة

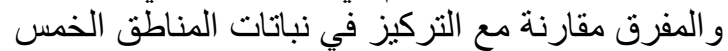

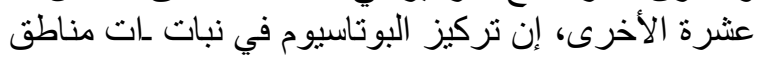

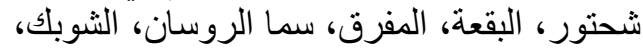

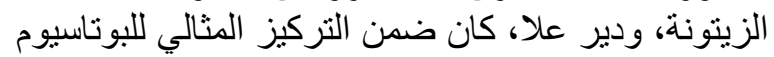

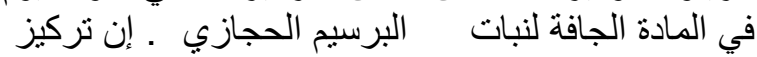

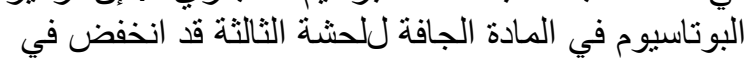

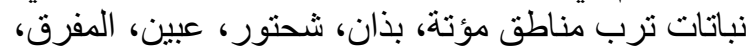

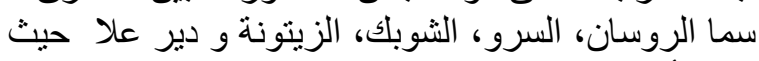

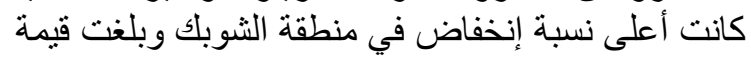

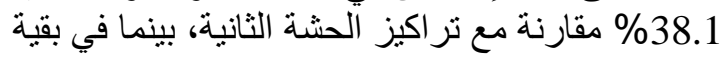

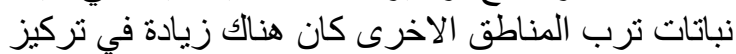

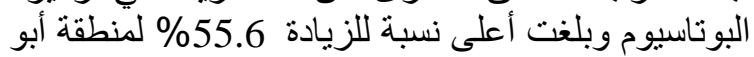

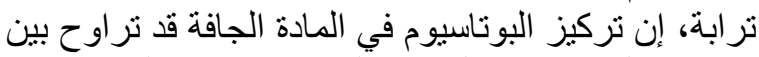

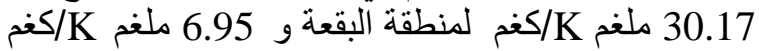
لمنطقة السرو ـ لقد نم إثبات الفروقات البفات المعنوية لتركيز

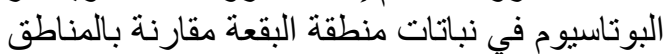

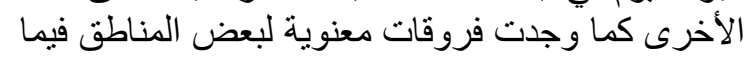

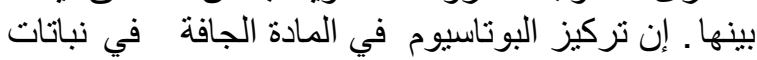

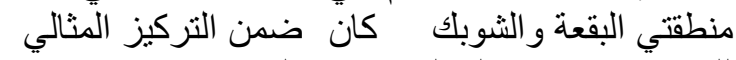

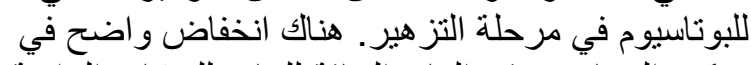

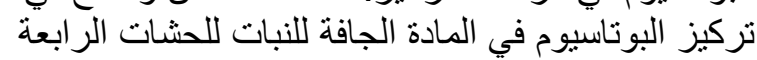

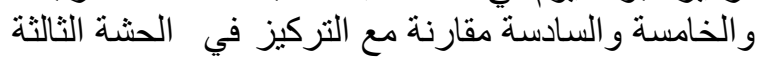

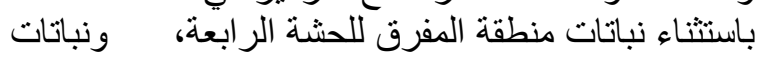

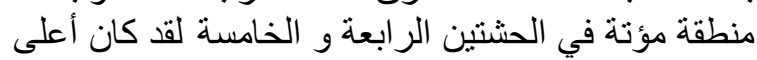
تركيز للبوتاسيوم في نباتات منطقة المفرق للحشئ الحنات

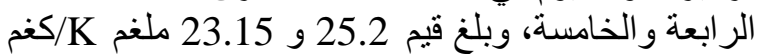

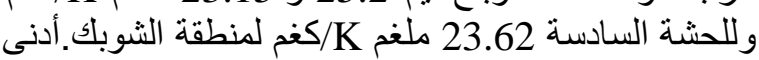

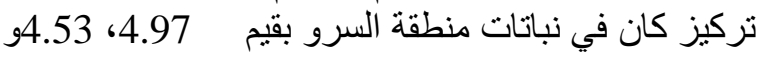

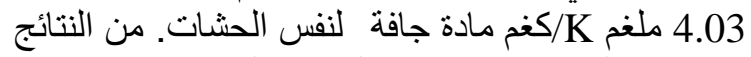
وجدت هناك فروقات معنوية لتركيز البوتاسيوم في نباتات منات

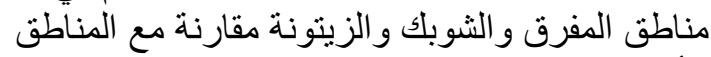
يبين الجدول ( 4) تركيز ات البوتاسيوم الذائب

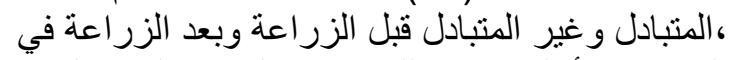

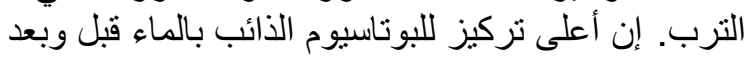

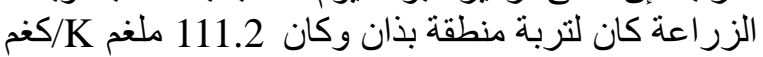

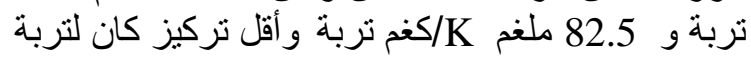

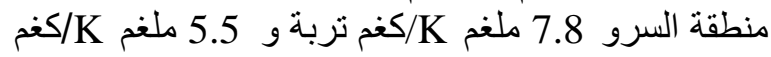
تربة وبلغت النسبة المئوية لنقصان تركيز البونة البوتاسيوم

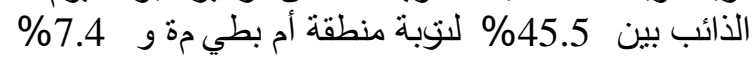

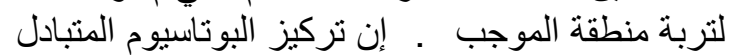
Exchangeable K

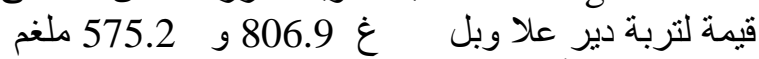

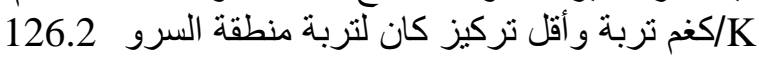

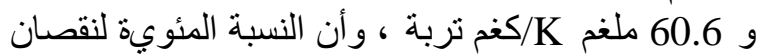

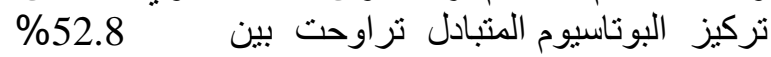
لتربـة سما الروسان و 10.1 \% لتربيز لتربة الربة ما قبل وبعد exchangeable K

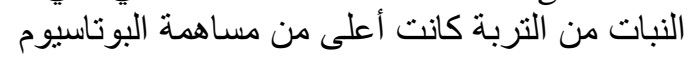

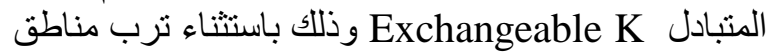

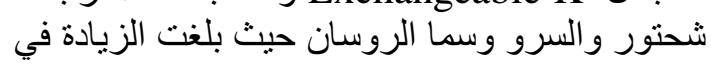

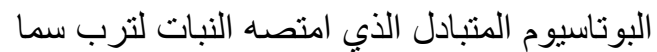

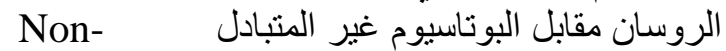
exchangeable K

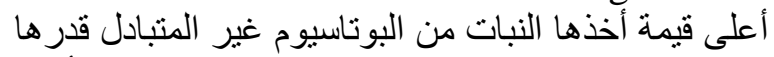

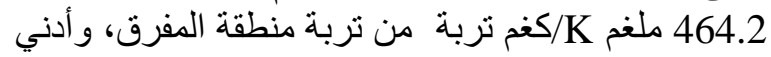

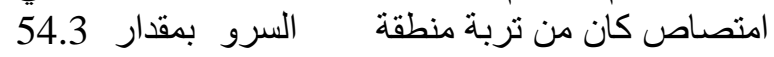

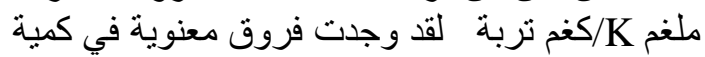

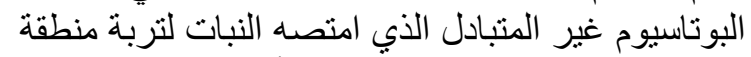

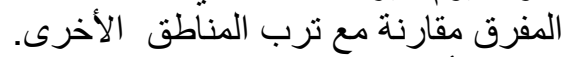

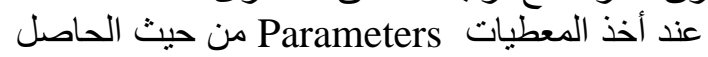

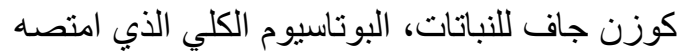

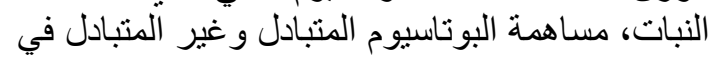

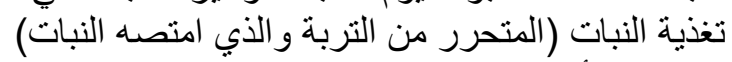
فإنه يمكن أن تقسم التربة لكافة المناطق إلى إلى مجمو عنين النين

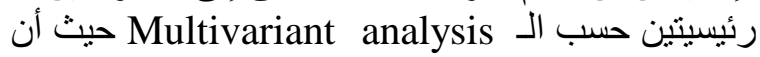

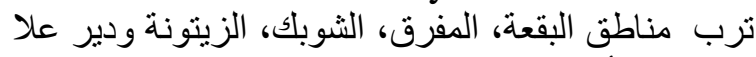

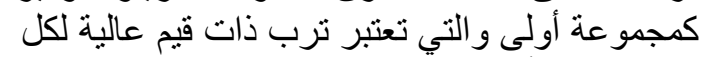

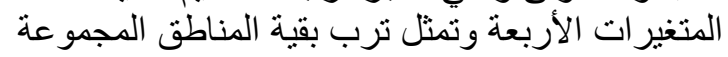

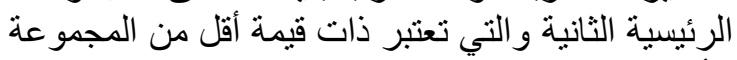

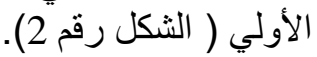

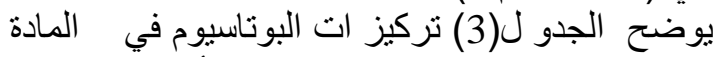

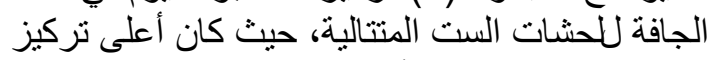

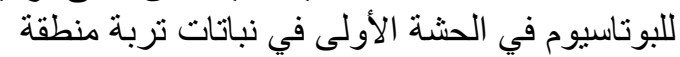

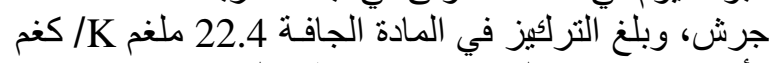

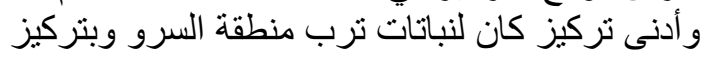

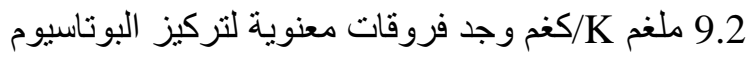

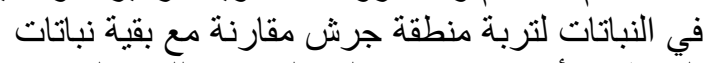

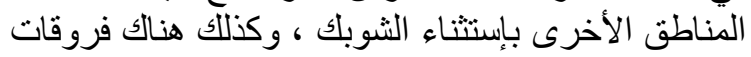

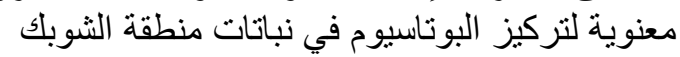

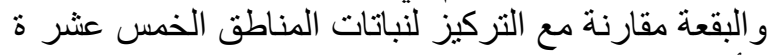

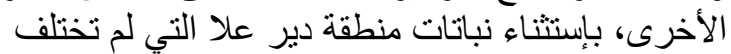
معنوياً عن نباتات منطقة البقعة. إن تركيز البوتاسيوم في النباتات كان أقل من التبات التركيز

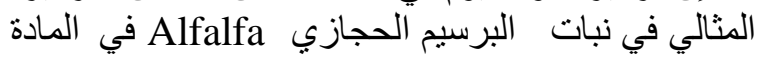

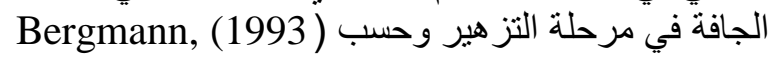

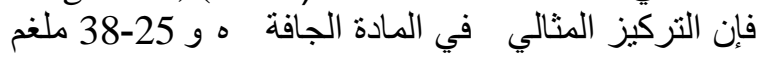

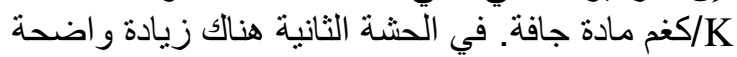
في تركيز البوتاسيوم في النبات لجميع المناطق بانية باستنثاء

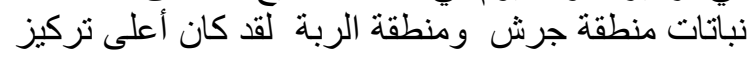

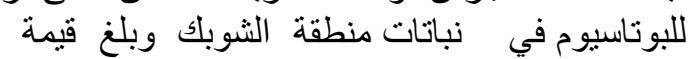

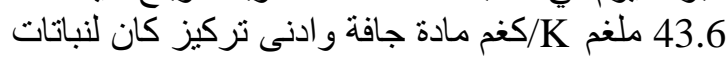

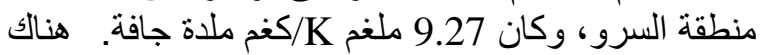

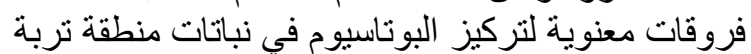

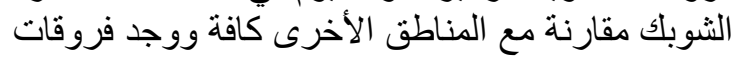




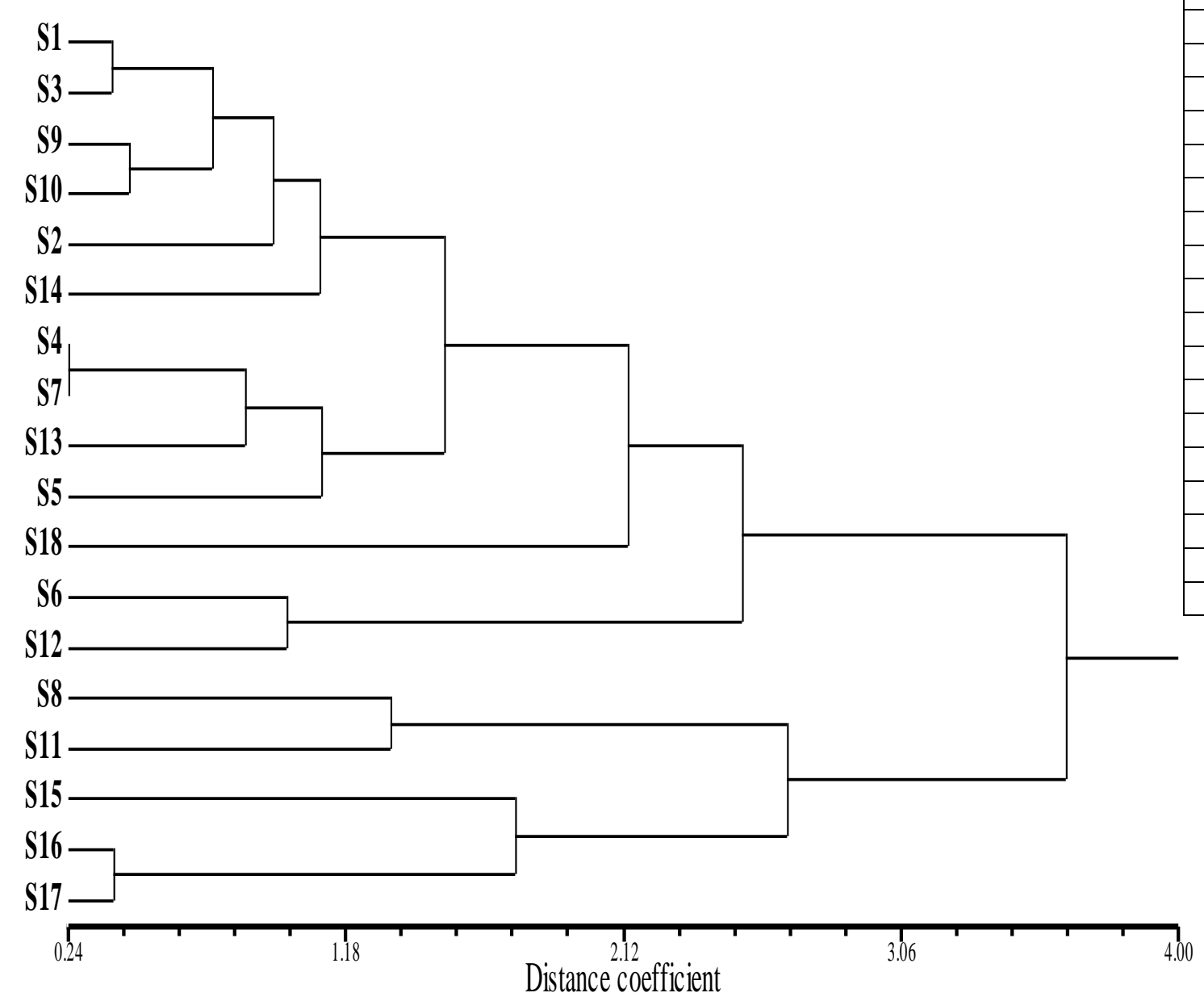

\begin{tabular}{|c|c|c|}
\hline المنطقة & $\begin{array}{l}\text { Location of } \\
\text { collected soils }\end{array}$ & Abbr \\
\hline مؤتتة & Mutah & S1 \\
\hline الحربية & alharbih & S2 \\
\hline الربة & alrabah & S3 \\
\hline ام بطيمة & um abtamah & S4 \\
\hline بذان & Bathan & S5 \\
\hline شحتور & Shahtur & S6 \\
\hline عبين & Abien & S7 \\
\hline البقعة & Albagah & S8 \\
\hline ابو ترابة & Abu Trabah & S9 \\
\hline الموجب & Almugab & S10 \\
\hline المفرق & Almafrag & S11 \\
\hline سما الروسان & Sama Alrusan & S12 \\
\hline الكورة & Alkurah & S13 \\
\hline السرو & Alsuraw & S14 \\
\hline الشوبك & Alshubak & S15 \\
\hline الزيتونة & Alzaitunah & S16 \\
\hline دير علا & Deer Alah & S17 \\
\hline جرش & Jarash & S18 \\
\hline
\end{tabular}

Fig. (2:) Clustering analysis of different soils at different locations. 
Table (3): Concentrations of $\mathrm{K}$ ( $\mathrm{mg} \mathrm{Kg}^{-1}$ dry weight) in alfalfa cuts.

\begin{tabular}{|c|c|c|c|c|c|c|c|c|}
\hline المنطقة & Location & Soil No. & Cut 1 & Cut 2 & Cut 3 & Cut 4 & Cut 5 & Cut 6 \\
\hline مؤتة & Mutah & 1 & 13.63 ghi & $14.93 j$ & 12.27h & 14.07ef & $15.57 \mathrm{~cd}$ & 9.18defg \\
\hline الحربية & alharbih & 2 & $11.71 \mathrm{ij}$ & $14.36 j$ & $16.73 \mathrm{~g}$ & $9.41 \mathrm{~g}$ & $9.99 f$ & 6.32gh \\
\hline الربة & alrabah & 3 & 17.20 de & $14.48 j$ & $16.96 \mathrm{~g}$ & $11.95 \mathrm{fg}$ & 12.62def & 6.62egh \\
\hline ام بطيمة & um abtamah & 4 & $15.10 \mathrm{fg}$ & $16.35 i j$ & 19.46efg & 16.71cde & 14.93cde & 8.11defgh \\
\hline بذان & Bathan & 5 & 16.6 def & $23.66 f g$ & 20.35fde & 19.69bc & $15.69 \mathrm{~cd}$ & 14.37bc \\
\hline شتور & Shahtur & 6 & $13.76 \mathrm{gh}$ & $25.73 \mathrm{ef}$ & 21.09def & 16.67cde & 12.89cdef & 10.46cdefg \\
\hline عبين & Abien & 7 & 15.5 efg & 21.42gh & $16.82 \mathrm{~g}$ & 16.8cde & 11.02ef & 10.94cde \\
\hline البقعة & Albagah & 8 & 20.01 bc & 29.21cd & $30.17 a$ & 22.7ab & $15.69 \mathrm{~cd}$ & $17.34 b$ \\
\hline ابو ترابة & Abu Trabah & 9 & 11.52 j & 14.03j & 21.84cde & 11.71fg & 13.92cdef & 6.34gh \\
\hline الموجب & Almugab & 10 & 16.82def & $20.1 \mathrm{~h}$ & $23.19 \mathrm{~cd}$ & 15.22def & $9.81 f$ & 10.64cdfe \\
\hline المفرق & Almafrag & 11 & 17.2de & $37.24 b$ & $24.55 \mathrm{bc}$ & $25.16 \mathrm{a}$ & $23.15 a$ & $22.84 a$ \\
\hline سما الروسـان & Sama Alrusan & 12 & $15.22 \mathrm{fg}$ & 27.89de & $23.36 \mathrm{~cd}$ & 17.93cde & 17.04bc & $16.02 b$ \\
\hline الكورة & Alkurah & 13 & 13.03hij & $15.24 \mathbf{j}$ & 18.33fg & 11.1fg & 11.78def & 7.13efgh \\
\hline السرو & Alsuraw & 14 & $9.2 \mathrm{kl}$ & $9.27 k$ & $6.95 i$ & 4.97h & $4.53 \mathrm{~g}$ & 4.03h \\
\hline الشوبك & Alshubak & 15 & $21.17 \mathrm{ba}$ & 43.59a & $26.98 b$ & 24.13a & 20.83ab & $23.62 a$ \\
\hline الزيتونة & Alzaitunah & 16 & 16.48def & 34.85b & 19.6efg & $16.35 \mathrm{cde}$ & $15.05 c d e$ & 11.41cd \\
\hline دير علا & Deer Alah & 17 & $18.43 \mathrm{~cd}$ & $31.94 c$ & 24.46bc & 24.13a & 20.79ab & 18.27b \\
\hline جرش & Jarash & 18 & 22.39 a & 19.02hi & 21.75cde & 19.04bcd & 20.83ab & 11.02cde \\
\hline
\end{tabular}


Table (4): Concentration of exchangeable and non-exchangeable $K$ in soil before and after planting and the percentage of reduction.

\begin{tabular}{|c|c|c|c|c|c|c|c|c|c|c|c|}
\hline \multirow{3}{*}{ المنطقة } & \multirow{3}{*}{ Location } & \multirow{3}{*}{$\begin{array}{l}\text { Soil } \\
\text { Nr. }\end{array}$} & \multicolumn{3}{|c|}{ Wat. Sol. K } & \multicolumn{3}{|c|}{ Exch. K } & \multicolumn{3}{|c|}{ Non-exch. K } \\
\hline & & & \multicolumn{9}{|c|}{ mg kg-1 soil } \\
\hline & & & Initial & Final & $\begin{array}{c}\text { Reduction } \\
(\%)\end{array}$ & Initial & Final & $\begin{array}{c}\text { Reduction } \\
(\%)\end{array}$ & Initial & Final & $\begin{array}{c}\text { Reduction } \\
(\%)\end{array}$ \\
\hline مؤتة & Mutah & 1 & 10.9 & 8.7 & 20.1 & 271.5 & 235 & 13.4 & 928.3 & 758.3 & 18.3 \\
\hline الحربية & alharbih & 2 & 14.9 & 9.1 & 38.9 & 305.3 & 231 & 24.3 & 1443.7 & 1236 & 14.3 \\
\hline الربة & alrabah & 3 & 17.8 & 13.9 & 21.9 & 446.4 & 401 & 10.1 & 1416 & 1242 & 12.3 \\
\hline ام بطيمة & um abtamah & 4 & 12.3 & 6.7 & 45.5 & 363.9 & 268.7 & 26.2 & 1417.2 & 1357.3 & 4.2 \\
\hline بذان & Bathan & 5 & 111.2 & 82.5 & 25.8 & 282.9 & 220 & 22.2 & 933.2 & 680 & 27.1 \\
\hline شحتور & Shahtur & 6 & 14.5 & 8.5 & 41.3 & 444 & 303 & 31.7 & 1126.8 & 1010 & 10.4 \\
\hline عبين & Abien & 7 & 11.9 & 7.6 & 36.1 & 425.9 & 341.6 & 19.8 & 1320.9 & 1188 & 10.4 \\
\hline البقعة & Albagah & 8 & 16.4 & 14.9 & 9.1 & 446.9 & 299 & 33 & 1273.1 & 933 & 26.7 \\
\hline ابو ترابة & Abu Trabah & 9 & 19.9 & 17.8 & 10.5 & 283.6 & 205.6 & 27.5 & 558.4 & 470 & 15.8 \\
\hline الموجب & Almugab & 10 & 27 & 25 & 7.4 & 425.1 & 347 & 18.3 & 1540.5 & 1040 & 32.4 \\
\hline المفرق & Almafrag & 11 & 69.9 & 47.4 & 32.1 & 535.7 & 398 & 25.7 & 1669.9 & 1532 & 8.2 \\
\hline سما الروسـان & Sama Alrusan & 12 & 25 & 14.3 & 42.8 & 487.4 & 229.8 & 52.8 & 1008 & 785.3 & 11 \\
\hline الكورة & Alkurah & 13 & 13.4 & 10.4 & 22.3 & 440.4 & 380 & 13.7 & 1371.3 & 1182 & 13.5 \\
\hline السرو & Alsuraw & 14 & 7.8 & 5.5 & 29.4 & 126.2 & 60.6 & 40.4 & 344.3 & 298.5 & 13.8 \\
\hline الشوبك & Alshubak & 15 & 90.1 & 57.7 & 35.9 & 600.4 & 335 & 44.2 & 877.6 & 757.3 & 13.3 \\
\hline الزيتونة & Alzaitunah & 16 & 20.7 & 12.9 & 37.6 & 480.5 & 314 & 34.6 & 1409.6 & 1160.6 & 13.7 \\
\hline دير علا & Deer Alah & 17 & 49.4 & 29.9 & 39.4 & 806.9 & 575.2 & 28.7 & 1395.3 & 1237 & $\mathbf{1 7 . 6}$ \\
\hline جرش & Jarash & 18 & 11.7 & 7.5 & 35.8 & 491.7 & 359 & 26.9 & 1538.7 & 1352.5 & 11.3 \\
\hline
\end{tabular}


الكلسية، ان مقدار المادة الجافة للذرة الصفر اء نراو ح بين

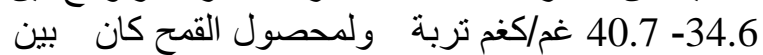

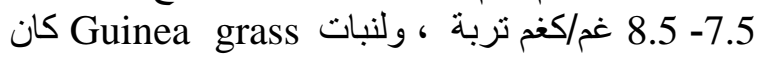

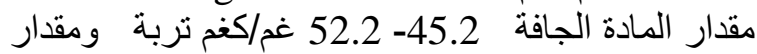

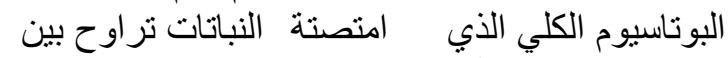

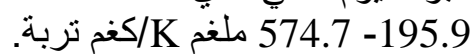

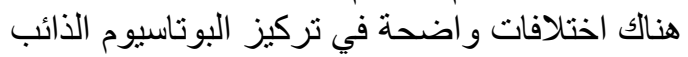

في محلول التربة، المتبادل وغير في المتبادل soluble, Exchangeable and Nonexchangeable K

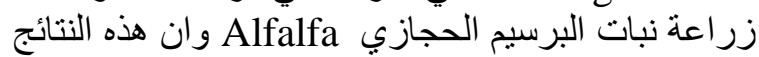
تطابق نتائج در اسات سابقة على الترب الكلسية في مناطق لنق

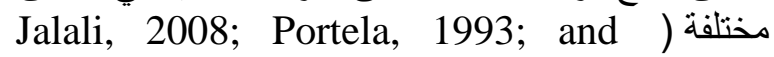

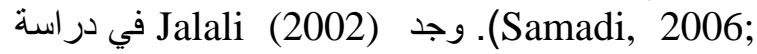

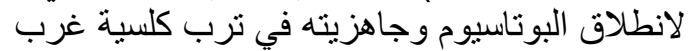

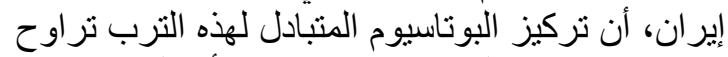

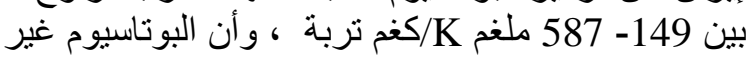

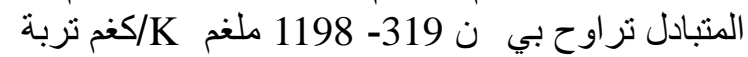

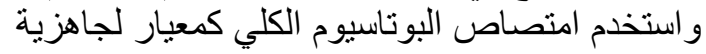

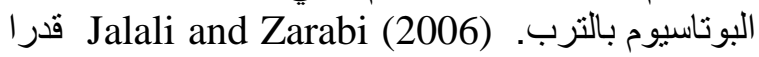

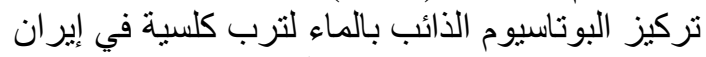

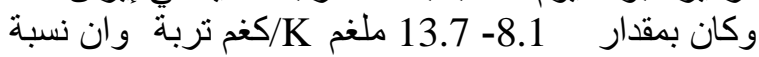

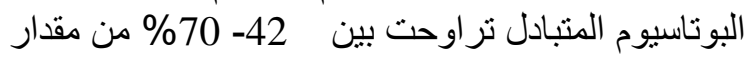

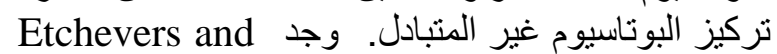

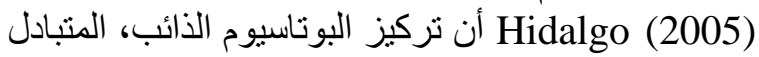

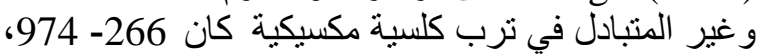

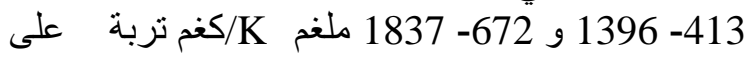

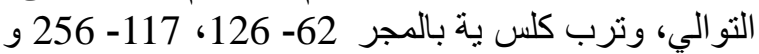

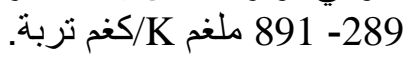
هناك نقص واضح في تركيز البوتاسيوم الذائب،

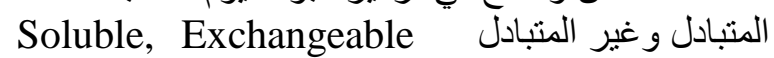
and Non-exchangeable K

البرسيم Alfalfa و أن أعلى نقصان كان في نركيز

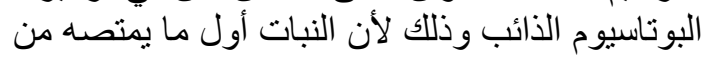

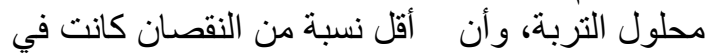

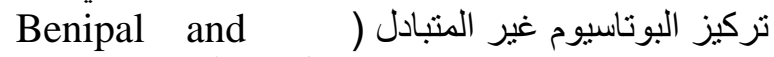

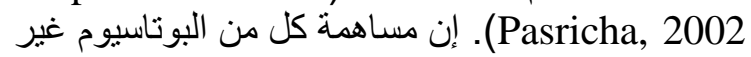

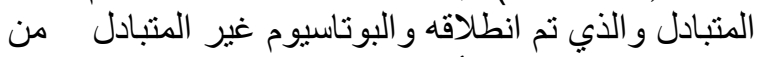

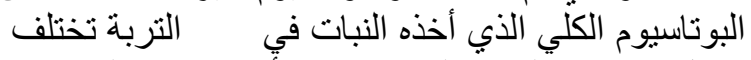

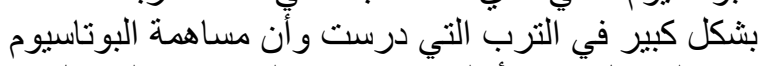

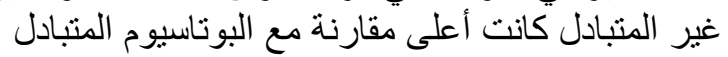

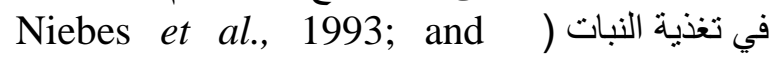

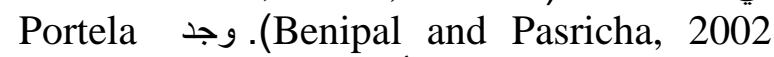
(1993) في الترب الكلسية أن نسبة انطلاق البوتاسيوم

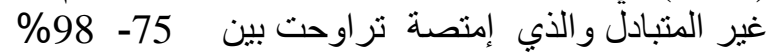

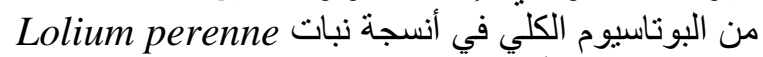
و ryegrass و أن كميـة البوتاسيوم أنبات الكلي الذي امتصد
Non- الزراعـة. إن البوتاسيوم غيـر المتبــادل exchangeable K K

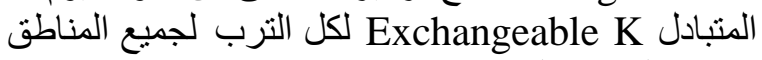

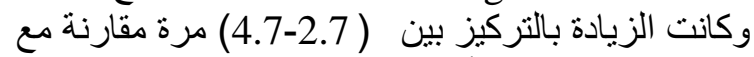

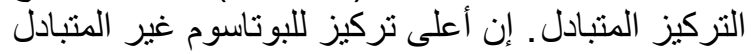

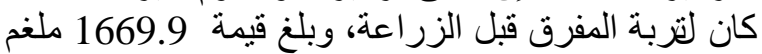

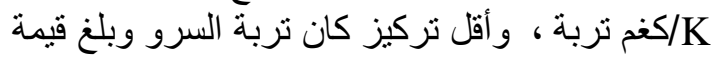
344.3 ملغم 1532

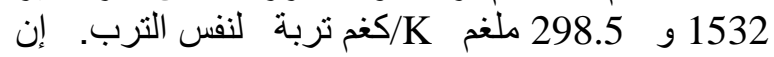

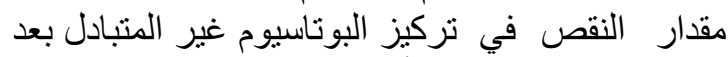

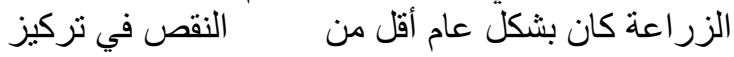

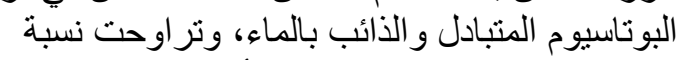

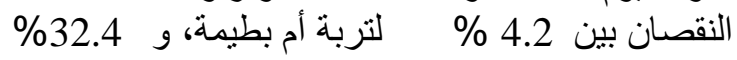

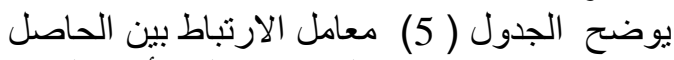

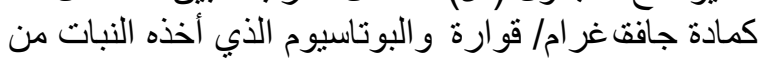

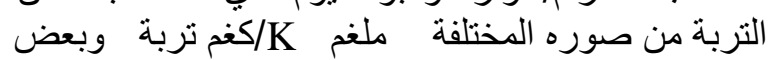
صفات التربة هناك علاقة معنو التية علية عالية بين المادة الجافية

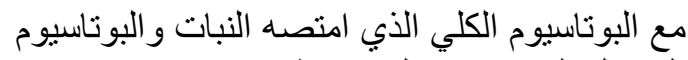
المنبادل البدائي بمعامل أرتباط مساو النيا

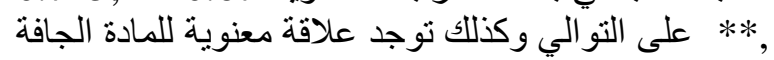

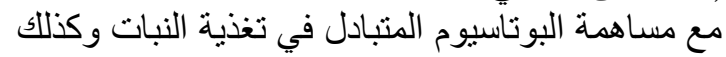

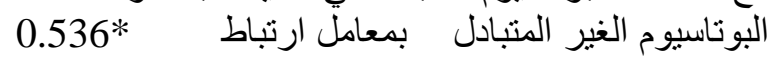
0.589,

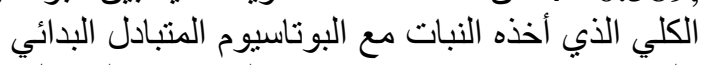

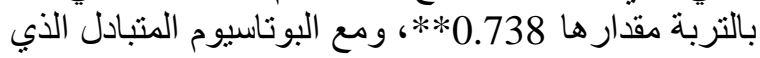

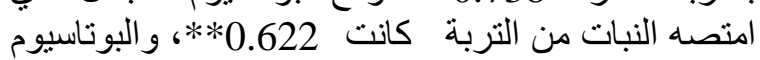
غير المتبادل الذي أخذة النبات من التربة .**0.884

\section{4.}

توضح النتائج الفروقات في كمية الحاصل التراكمي التئي

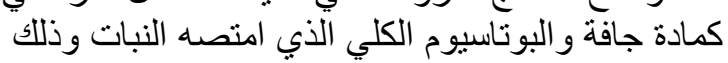

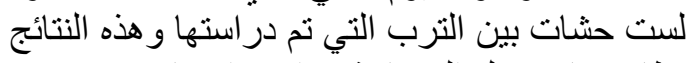

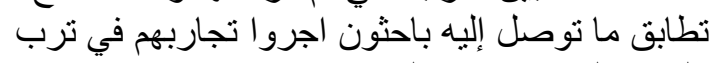

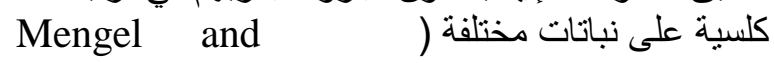
Rahmatullah, 1994; Zubillaga and Conti, (1996; and Sharma et al., 2006 Havlin and Westfall (1985)

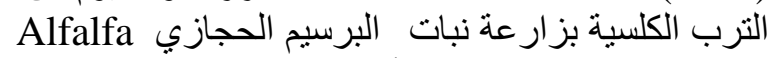
وبعد ست عشرة حشة وجدا أن مقدار المادة الجافة للنبات

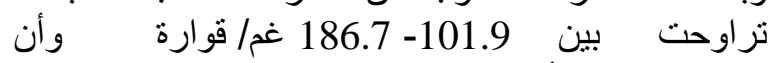

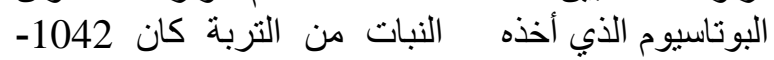

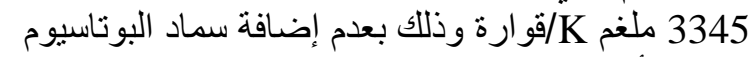

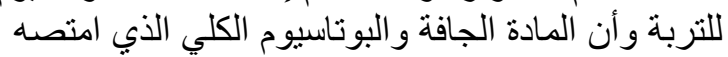

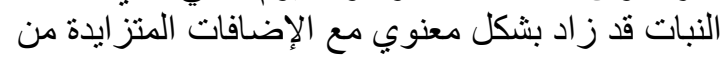

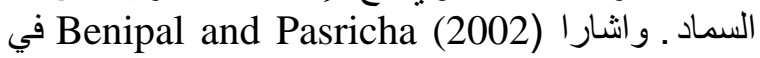
دراسة لهما لانطلاق البوتاسيوم غير المتبادل في الترب في 


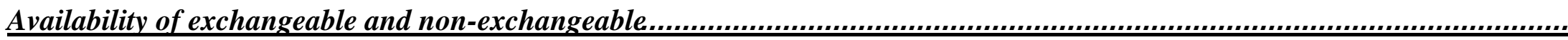

Table (5): Correlation coefficient among different parameters.

\begin{tabular}{|c|c|c|c|c|c|c|c|c|c|c|c|c|}
\hline & Silt & Clay & pH & O.M. & $\mathrm{CaCO}_{3}$ & CEC & $\begin{array}{l}\text { Dry } \\
\text { matter }\end{array}$ & $\begin{array}{l}\text { Total K- } \\
\text { upt. }\end{array}$ & $\begin{array}{l}\text { Ex.K- } \\
\text { upt. }\end{array}$ & $\begin{array}{l}\text { Non-ex. } \\
\text { K-upt }\end{array}$ & $\begin{array}{l}\text { Int.ex,K } \\
\text { soil }\end{array}$ & $\begin{array}{l}\text { Int.Non- } \\
\text { ex.K soil }\end{array}$ \\
\hline Silt & 1 & 0.116 & -0.043 & $-0.475 *$ & 0.442 & 0.012 & -0.137 & 0.117 & 0.125 & 0.075 & 0.244 & 0.404 \\
\hline Clay & & 1 & 0.266 & -0.052 & -0.346 & $0.647 * *$ & 0.647 & -0.244 & 0.079 & -0.361 & 0.093 & 0.058 \\
\hline pH & & & 1 & -0.423 & 0.159 & -0.155 & -0.175 & -0.362 & -0.106 & -0.39 & -0.141 & -0.161 \\
\hline O.M. & & & & 1 & -0.418 & 0.272 & $0.654 * *$ & $0.59 * *$ & 0.448 & $0.469 *$ & 0.431 & 0.021 \\
\hline $\mathrm{CaCO3}$ & & & & & 1 & $-0.54 *$ & -0.122 & 0.157 & -0.096 & 0.266 & 0.129 & 0.399 \\
\hline CEC & & & & & & 1 & $0.452 *$ & 0.152 & 0.12 & 0.113 & 0.313 & 0.09 \\
\hline Dry matter & & & & & & & 1 & $0.725 * *$ & $0.536 *$ & $0.589 *$ & $0.672 * *$ & 0.242 \\
\hline Toltal K-upt. & & & & & & & & 1 & $0.622 * *$ & $0.884 * *$ & $0.738 * *$ & 0.291 \\
\hline Ex.K-upt. & & & & & & & & & 1 & 0.186 & $0.586 * *$ & -0.068 \\
\hline Non-ex.K-upt. & & & & & & & & & & 1 & 0.58* & 0.408 \\
\hline Int.ex.K Soil & & & & & & & & & & & 1 & $0.537 *$ \\
\hline Int. Non-ex.K & & & & & & & & & & & & \\
\hline Soil & & & & & & & & & & & & 1 \\
\hline
\end{tabular}

O. M., Organic Matter; Int.ex.K, initial exchangeable K; Int. Non-ex. K, initial Non-Exchangeable K. 


$$
\begin{aligned}
& \text { البدائي بالتربة وبين الحاصل الكلي لنبات ال برسيخ } \\
& \text { Alfalfa }
\end{aligned}
$$

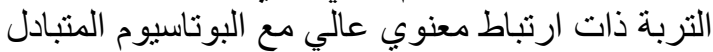

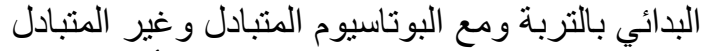

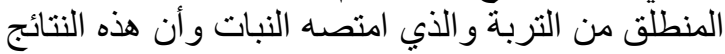

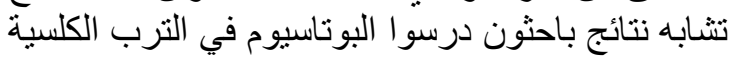

Zubillaga and Conti, 1996; and Jalali and )

Sharma et al. (2006) (Zarabi, 2006 أن هناك علاقة ارتباط معنوية بين البوتاسيوم الكلي الكنطلق في التربة ومحتوى كل من الطين و والغرين البرني

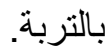

\section{5}

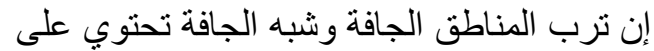

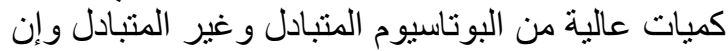

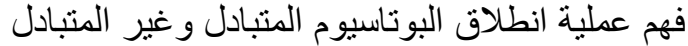

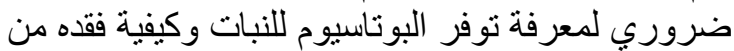

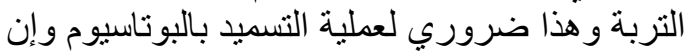

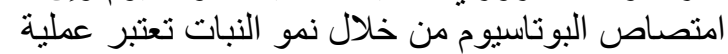

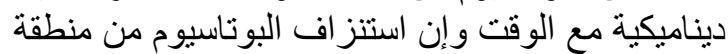

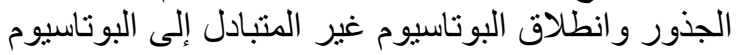

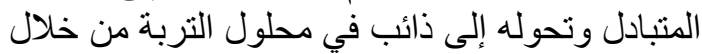

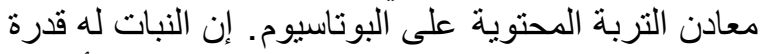

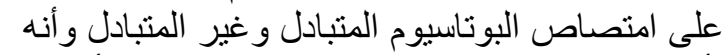

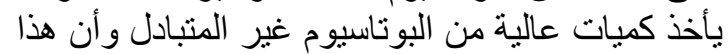

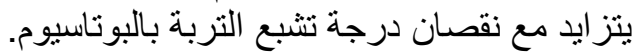

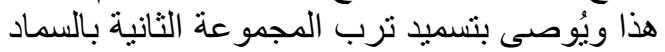

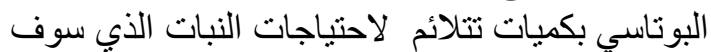

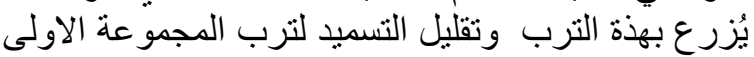

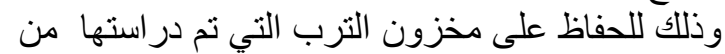

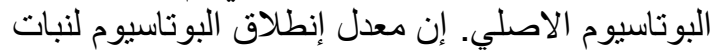

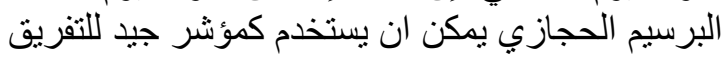

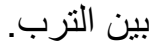

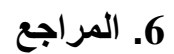

Benipal D. S. and Pasricha N. S. (2002). Nonexchangeable $\mathrm{K}$ release and supplying power of Indo-Gangetic alluvial soils. Geoderma,, 108:197206.

Bergmann W. (1993). Ernaehrungsstoerungen bei Kulturpflanzen. Gustav Fischer Verlag. Sttuttgart. 835. pp.

Binet P., Elguessabi L. and Salette J. (1984). The potassium status of soils: Significance of the Italian ryegrass test. Fert. Res., 5: 393-402.

Bouyoucos G. J. (1951). Recalibration of hydrometer method of making

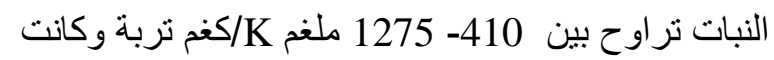

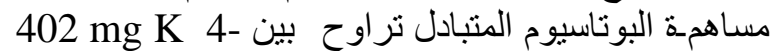
ئ kg- ${ }^{1}$ soil

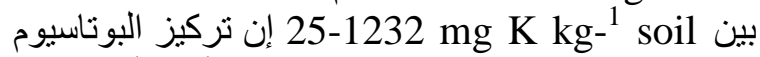
في المادة الجافة للنبات كان في الحشة الأولى أقل من التوني

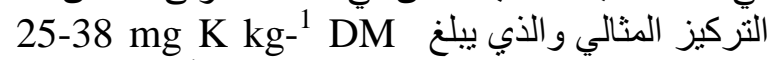
حسب (Bergmann, 1993) وذللك لجميع أنواع التئ الترب،

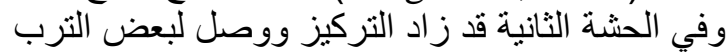

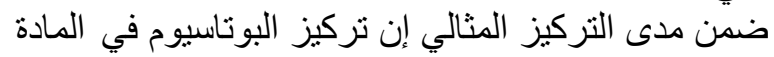

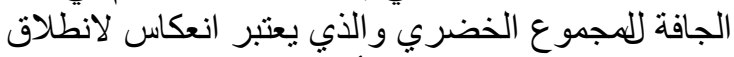

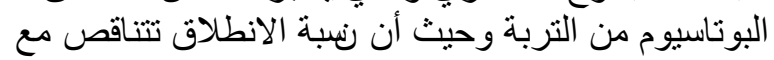

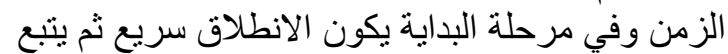

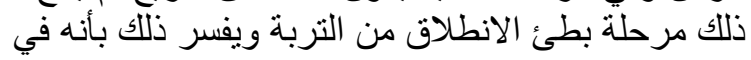

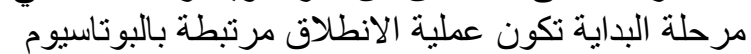

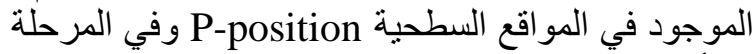

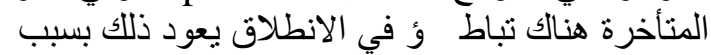

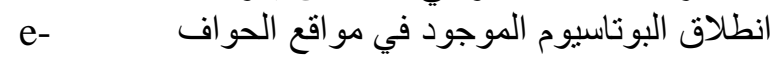
position

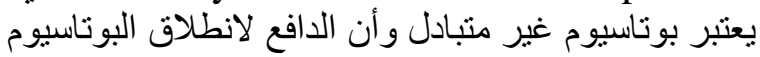

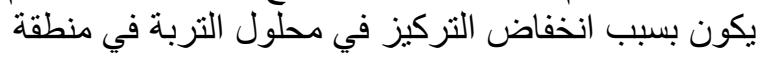

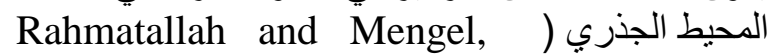
(2000; and Jalali, 2008 نظهر النتائج العلاقة المعنوية العالية بين المادة الجافة

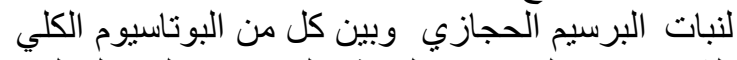

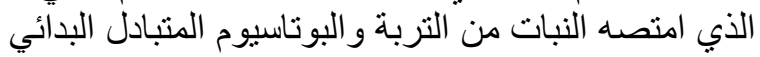

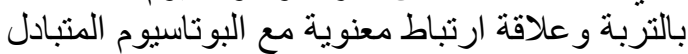

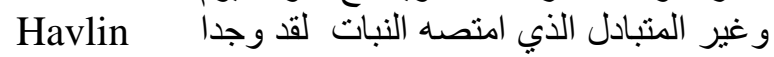
and Westfall (1985) مقدار ها ** r r =0.87 بين البوتاسيوم المتبادل

mechanical analysis of soil. Agron. J., 43: 434-435.

Cox A. E., Joern B. C., Brouder S. M. and Gao D. (1999).Plant-available potassium assessment with a modified sodium tetraphenylboron method. Soil Sci. Soc. Am. J., 63: 902-911.

Dhillon S. K. and Dhillon K. S. (1990). Kinetiecs of release of nonexchangeable potassium by cationsaturated resins form red (alfisols), black (vertisols) and alluvial (Inceptisols) soils of India. Geoderma., 47: 283-300.

Etchevers J. D. and Hidalgo C. M. (2005). Dynamics of soil $\mathrm{K}$ release. Commu. Soil Sci. Plant anal., 36: 341-354. 
Havlin J. L. and Westfall D. G. (1985). Potassium release kinetics and plant response in calcareous soil. Soil Sci. Soc. Am. J. 49: 366-370.

Jalali M. (2002). Composition of irrigation water in west of Iran. $17^{\text {th }}$ World Congress of Soil Science in Bangkok. Thailand. Vol. 14-21, PP. 2184-1August.

Jalali M. (2005). Release kinetics of nonexchangeable potassium in Calcareous soils. Commun. Soil Sci. Plant Anal., 36: 1903-1917.

Jalali M. (2006). Kinetics of nonexchangeable potassium release and availability in some calcareous soils of western Iran. Geoderma., 135: 63-71.

Jalali M. (2008). Effect of sodium and magnesium on kinetics of potassium release in some calcareous soils of western Iran. Geoderma., 145: 207215.

Jalali M. and Zarabi M., (2006). Kinetics of nonexchangeable-potassium relrease and plant response in some calcareous soils. J. Plant Nutr. Soil Sci., 169: 196-204.

Maclean E. O. and Watson M .E. (1985). Soil measurements of plant-available potassium. In Potassium in Agriculture. Ed. R. D. Munson. p. 278-309. Soil Sci. Soc. Am., Madison. WI.

Mengel K. (1985). Dynamics and availability of major nutrients in soils. Adv. Soil Sci., 2: 65-133.

Mengel K. and Rahmatullah B. Z. (1994). Exploitation of potassium by various crop species from primary minerals in soils rich in micas. Biol. Fertil. Soils., 17: 75-79.

Mengel K. and Uhlenbecker K. (1993). Determination of available interlayer potassium and its uptake by ryegrass. Soil Sci. Soc. Am. J., 57: 761-766.

Niebes J. F., Dufey J. E., Jaillard B. and Hinsinger P. (1993). Release of nonexchangeable potassium from different size fractions of two highly Kfertilized soils in the rhizosphere of rape (Brassica napus cv Drakkar) . Plant and Soil., 155/156 : 403-406.

Øgaard A. F. and Krogstad T. (2005). Release of interlayer Potassium in Norwegian grassland soils. J. Plant Nutr. Soil Sci., 168: 80-88.

Portela E. A.C. (1993). Potassium supplying capacity of northeastern Portuguese soils. Plant and Soil., 154: 13-20.

Rahmatullah B. Z., and Mengel K. (2000). Potassium release from mineral structures by $\mathrm{H}^{+}$ion resin. Geodema., 96: 291-305.

Rahmatullah B. Z., Shaikh M. A. G. and Salim, M. (1994). Bioavailable potassium in river-bed sediments and release of interlayer Potassium in irrigated arid soils. Soil Use and Manag., 10: 43-46.

Rowell D. L. (1994). Soil Science: Methods and Applications. Springer, Berlin.

Samadi A. (2006). Potassium exchange isotherms as a plant availability index in selected calcareous soils of western Azarbaijan province, Iran. Turk. J. Agric. For., 30: 213-222.

Sharma B. D., Mukhopadhyay S. S. and Sawhney J. S. (2006). Distribution of potassium fractions in relation to landforms in a Himalayan catena. Arch. Agro. and Soil Sci., 52: 469476.

Sparks D. L. (1987). Potassium dynamics in soils. Adv. Soil Sci., 6: 1-63.

Sparks D. L. and Huang P. M. (1985). Physical chemistry of soil potassium. In Munson, R. D., et al. (eds.): Potassium in Agriculture. American Society of Agronomy, Madison, Wisconsin, p. 201-276.

Zubillaga M. M. and Conti E. (1996). Availability of exchangeable and nonexchangeable $\mathrm{K}$ in Argentine soils with different mineralogy. $\mathrm{Z}$. Pflanzenernaehr. Bodenk., 159: 149153. 